Reprod. Nutr. Dévelop., 1983, 23 (2 A), 161-173.

\title{
Digestion des protéines du lait dans la caillette du veau préruminant. Evacuation gastrique après un repas d'épreuve
}

\author{
J.-P. PÉlissieR, P. GUILLOTEAU $\left({ }^{*}\right)$, G. BRULE $\left({ }^{* *}\right)$, R. TOULLEC $\left({ }^{*}\right)$ \\ Laboratoire de Biochimie et Technologie laitières, I.N.R.A., \\ 78350 Jouy-en-Josas. \\ (*) Station de Recherches zootechniques, I.N.R.A. \\ $\left.{ }^{* *}\right)$ Laboratoire de Recherches de Technologie laitière, I.N.R.A., \\ 65, rue de Saint-Brieuc, 35000 Rennes.
}

Summary. Digestion of milk protein in the abomasum of preruminant calf. Gastric evacuation after a test meal.

In this study, we used fasted, preruminant calves fitted with a double proximal cannula in the duodenum ; gastric evacuation was investigated by recovering products leaving the abomasum after a test meal. Five types of meal were given : whole milk, skimmed milk, casein solution in water, casein solution in a mineral medium simulating the permeate of highly-filtered milk, and whey. Gastric emptying of nitrogen was relatively slow. The coagulation of " milk diets" slowed down this emptying. Precipitation of " casein diets " at acid $\mathrm{pH}$ also slowed down emptying, but to a lesser degree. The "casein diets ", more proteolyzed than the "milk diets ", released larger amounts of non-protein nitrogen (NPN) into the intestine. Calcium emptying was highly related to NPN emptying, demonstrating the important role of acid peptides (phosphopeptides) from casein hydrolysis.

\section{Introduction.}

La première étape de la digestion des protéines a lieu dans I'estomac. La protéolyse gastrique se produit sous l'action des pepsines et, chez le jeune de certaines espèces, sous l'action de la chymosine (Hill, Noakes et Lowe, 1970 ; Foltmann et al., 1981). La dégradation in vitro des caséines par la chymosine et les pepsines bovines est assez bien connue (Delfour et al., 1965 ; Fox et Walley, 1971 ; Creamer, Mills et Richard, 1971 ; Hill, Lahav et Givol, 1974 ; Pélissier, Mercier et Ribadeau-Dumas, 1974 ; Visser et Slangen, 1977 ; Addeo, Pélissier et Chianese, 1980 ; Visser, 1981). En revanche, il existe peu de données concernant la digestion in vivo de protéines du lait dans l'estomac. En dehors de quelques études du contenu stomacal de rats nourris avec des lactoprotéines (Miranda et Pélissier, 1981 a, b ; 1982), les principaux travaux sur la digestion stomacale portent sur la vidange gastrique. Ainsi, un certain nombre de résultats sont connus 
chez le rat (Zebrowska, 1968 ; Buraczewski et al., 1971), le porc (Braude et al., 1970 a, b) et le veau (Toullec, Thivend et Mathieu, 1971; Ternouth et Roy, 1973 ; Guilloteau et al., 1975, 1979) alimentés avec du lait ou des régimes à base de caséine ou de protéines du lactosérum.

Dans le but de mieux connaître les mécanismes de la protéolyse, in vivo, des caséines dans la caillette du veau préruminant, nous avons entrepris d'étudier les produits peptidiques arrivant dans le duodénum proximal à la suite de l'ingestion d'un repas d'épreuve. Pour mettre en évidence les effets éventuels de la matière grasse, du lactosérum, des minéraux et de la coagulation, nous avons utilisé différents régimes : lait entier, lait écrémé, lactosérum, solution de caséine dans un milieu minéral simulant le perméat de lait ultrafiltré (Jenness et Koops, 1962) et solution de caséine dans l'eau. Dans une première étape, décrite dans cet article, nous avons précisé l'évacuation gastrique de ces régimes chez des veaux préalablement mis à jeun.

\section{Matériel et méthodes}

Alimentation et plan expérimental. - Les veaux recevaient habituellement un aliment d'allaitement contenant $25 \%$ de protéines apportées en totalité par du lait écrémé. Cet aliment, à une concentration de $125 \mathrm{~g}$ de matière sèche par $\mathrm{kg}$ de lait de remplacement, était distribué au seau deux fois par jour, les quantités offertes variant selon le poids des veaux de 3,5 à $5 \mathrm{~kg}$ par repas. La veille des prélèvements, le " lait » distribué au repas du matin était additionné de citrate de sodium $(11 \mathrm{~g} / \mathrm{kg})$, dans le but d'empêcher la coagulation sous l'action des enzymes et d'accélérer l'évacuation gastrique (Frantzen et al., 1973). Au repas du soir, le lait était remplacé par de l'eau. Ainsi, la caillette devait être quasiment vide au moment de la distribution du repas d'épreuve qui avait lieu le matin.

Chaque repas d'épreuve était constitué de $5 \mathrm{~kg}$ de l'un des régimes suivants : lait entier (A), lait écrémé (B), solution à $3 \%$ de caséine dans l'eau (C), solution à $3 \%$ de caséine dans un milieu minéral simulant le perméat de lait ultrafiltré (Jenness et Koops, 1962) (D) et lactosérum ( $E$ ). Les régimes $A$ et $B$ coagulaient, alors que les régimes $C$ et $D$, dépourvus de calcium, ne coagulaient pas mais précipitaient lorsque le $\mathrm{pH}$ approchait de 4,6. Le régime $\mathrm{E}$ a été utilisé pour identifier, dans les régimes $A$ et $B$, les peptides provenant du lactosérum par rapport à ceux provenant des caséines.

Tous ces régimes étaient préparés à partir du même lait provenant d'une seule vache, homozygote pour les 4 caséines $\left(\alpha_{\mathrm{s} 1}, \alpha_{\mathrm{s} 2}, \beta\right.$ et $\left.x\right)$. La caséine entière était isolée du lait écrémé par 3 précipitations à pH 4,6 à l'aide d'acide chlorhydrique $1 \mathrm{M}$. Après chaque précipitation, la partie insoluble était redissoute dans de l'eau par addition lente de soude $1 \mathrm{M}$ jusqu'à $\mathrm{pH} 7,0$. Finalement, la solution de caséine à $\mathrm{pH} 7,0$ était lyophilisée. Le lactosérum était préparé par précipitation des caséines du lait à $\mathrm{pH} 4,6$ à l'aide d'acide chlorhydrique $1 \mathrm{M}$, puis ajustement du $\mathrm{pH}$ 6,5 par addition de soude $1 \mathrm{M}$. Le lait entier, le lait écrémé et le lactosérum étaient conservés à $-18{ }^{\circ} \mathrm{C}$ jusqu'à utilisation. 
Animaux et prélèvements. - Deux veaux mâles de race Frisonne étaient munis d'une canule duodénale réentrante (Ash, 1962), la partie proximale étant placée $10 \mathrm{~cm}$ environ après le pylore. Les repas d'épreuve étaient administrés 15 jours au moins après la pose de la canule et au maximum une fois par semaine chez un même animal. Le premier veau recevait successivement les régimes $B, C, D, A, B, C, D, A, E$ et le second les régimes $B, C, D, A, B$. Au total, 14 repas d'épreuve ont ainsi été administrés.

Après chaque repas d'épreuve, les digesta quittant la caillette étaient recueillis en totalité, pendant 20 périodes réparties sur $7 \mathrm{~h}$ consécutives. La durée de chaque période était de $10 \mathrm{~min}$ pendant la première heure, de $15 \mathrm{~min}$ pendant la seconde et de $30 \mathrm{~min}$ pendant les 5 dernières. Afin de bloquer immédiatement toute réaction enzymatique, les béchers de collecte contenaient $100 \mathrm{ml}$ d'une solution d'acide trichloracétique (TCA) à $12 \%$. Dès la fin de chaque période, un volume de TCA à $24 \%$, égal à celui des digesta collectés, était ajouté dans le bécher, de façon à obtenir une concentration finale en TCA de $12 \%$. Les échantillons étaient conservés à $+4{ }^{\circ} \mathrm{C}$ jusqu'aux analyses.

Pendant les $7 \mathrm{~h}$ de collecte des digesta, un hydrolysat de lait écrémé était réintroduit dans le duodénum par la canule distale, à la température d'environ $37^{\circ} \mathrm{C}$, à un rythme voisin de celui de sortie des digesta. Cet hydrolysat, dont I'azote non protéique représentait environ $20 \%$ de l'azote total, était obtenu par action de présure liquide (présure Boll préalablement dialysée) sur du lait écrémé.

Analyses. - Chaque échantillon était centrifugé à $2000 \mathrm{~g}$ pendant $20 \mathrm{~min}$. Le surnageant était conservé en l'état jusqu'aux analyses. Le culot était remis en suspension dans l'eau, homogénéisé à l'aide d'un Ultra-Turrax (Garrant Lab., Paris, France) et son volume était ajusté à $250 \mathrm{ml}$ avec de l'eau. Les différents échantillons étaient conservés à $-18^{\circ} \mathrm{C}$. Le dosage de l'azote était effectué sur chacune des fractions suivant la technique de Kjeldahl, à l'aide d'un analyseur automatique Technicon, par dosage colorimétrique après minéralisation. L'azote dosé dans le surnageant correspondait à l'azote non protéique (NPN), celui mesuré dans le culot à l'azote protéique. Dans les surnageants obtenus avec les régimes contenant du calcium (régimes "laits": A et B) la teneur en calcium était déterminée par spectrométrie d'absorption atomique.

Les résultats obtenus ont été soumis à des analyses de variance et à des analyses en composantes principales afin d'essayer de mettre en évidence des différences entre les régimes.

\section{Résultats.}

La figure 1 indique les moyennes des quantités cumulées de matière fraîche recueillie pour chaque régime au cours du temps après le repas. Au bout de $7 \mathrm{~h}$, la quantité moyenne de matière fraîche ayant quitté la caillette est voisine de celle ingérée par l'animal lors du repas d'épreuve. On observe cependant une variabilité des résultats de l'ordre de $25 \%$ dans les répétitions effectuées avec un même régime. La figure 2 représente l'évolution du débit moven observé avec les 14 expériences. 


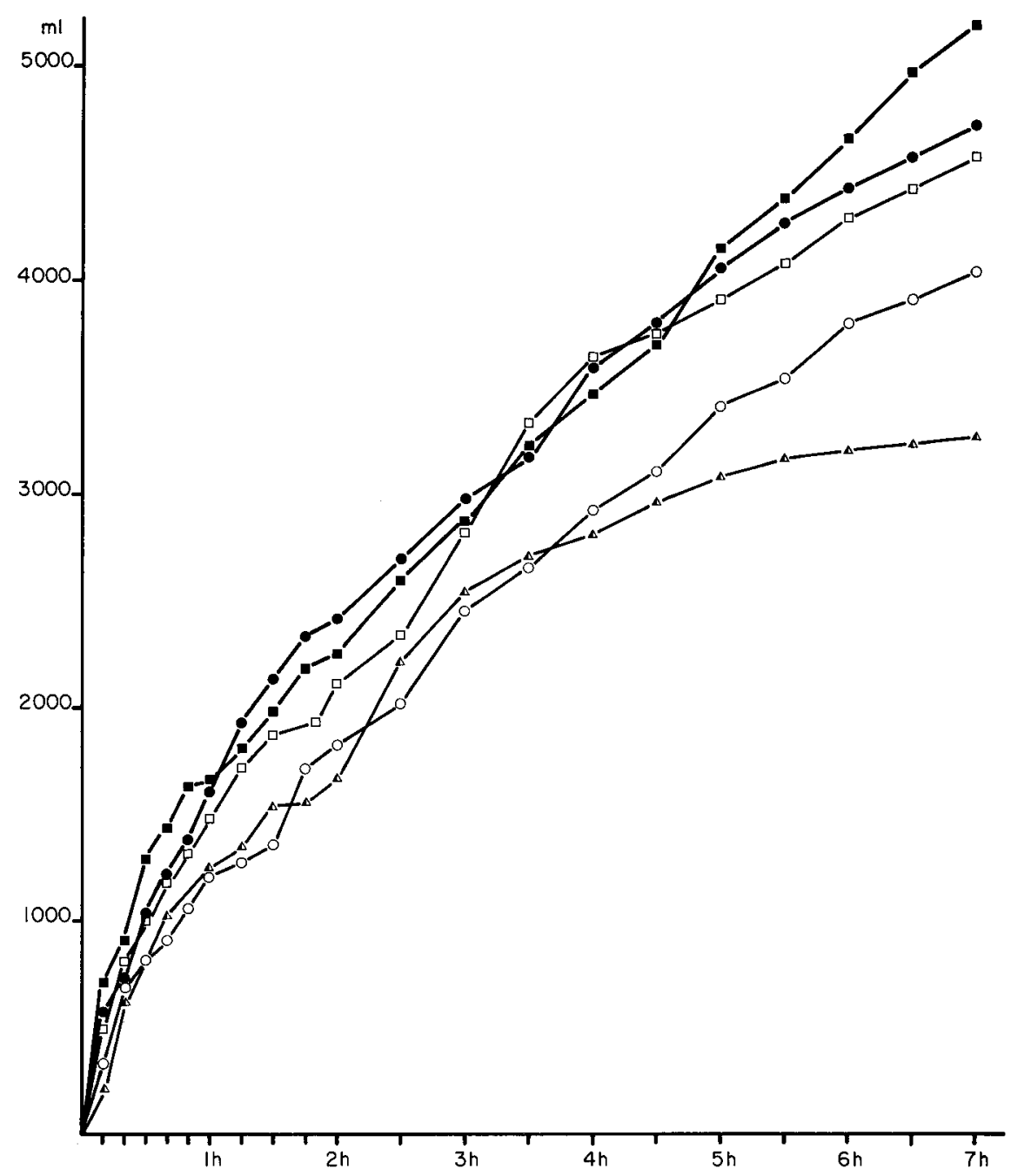

FIG. 1. - Evacuation gastrique de la matière fraîche (quantités cumulées par régime, en ml). - A Lait entier: B Lait écrémé; $O$ C Caséine dans l'eau; $\square$ D Caséine dans le milieu minéral ; $\Delta \mathrm{E}$ Lactosérum.

Les quantités cumulées d'azote total obtenues avec chacun des régimes au cours des 7 premières heures postprandiales (tabl. 1) montrent une dispersion beaucoup plus importante dans les cas des régimes $C$ et $D$ (variations allant jusqu'à $50 \%$ de la valeur moyenne) qu'avec les régimes $A$ et $B$ (variations inférieures à $20 \%$ ). Cependant, les résultats moyens observés (fig. 3) semblent indiquer un comportement différent des régimes " lait " $(A$ et $B$ ) qui coagulent par rapport aux régimes "caséines» ( $\mathrm{C}$ et $\mathrm{D}$ ) qui précipitent à $\mathrm{pH}$ inférieur à 5 et surtout par rapport au lactosérum (régime E) qui ne subit pas ce type de modifi- 


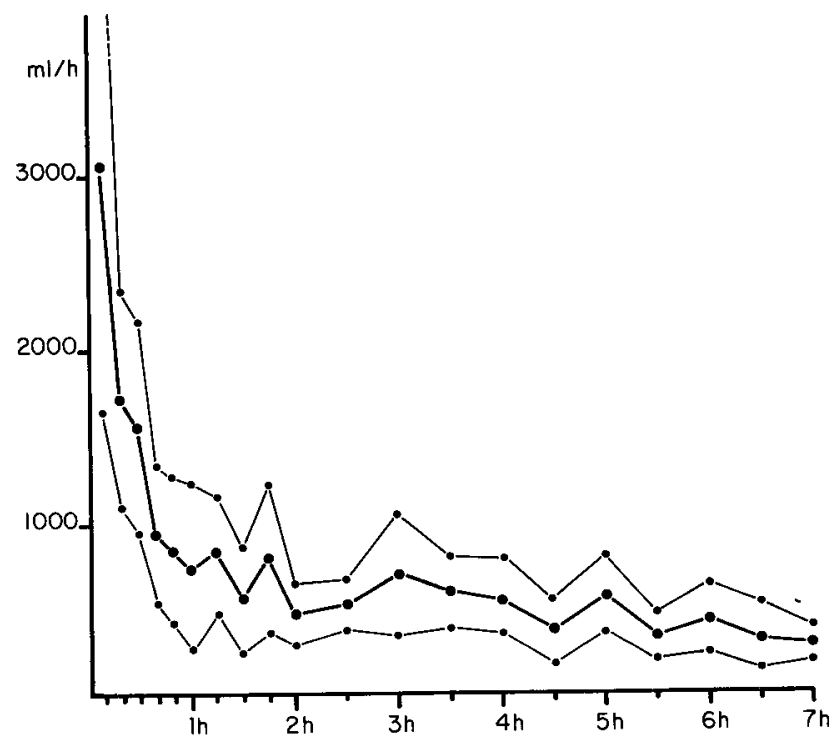

FIG. 2. - Evacuation gastrique de la matière fraiche $(\mathrm{ml} / \mathrm{h})$.

débits movens des 14 repas d'épreuve: pour $p=5 \%$.

TABLE 1

Evacuation gastrique de l'azote total

(quantités cumulées moyennes par régime et écarts-types de l'échantillon en mg)

\begin{tabular}{|c|c|c|c|c|c|}
\hline Durée & Régime A & Régime $B$ & Régime C & Régime D & Régime $E$ \\
\hline $10 \mathrm{~min}$ & $583(34,9)$ & $945(418,2)$ & $205(77,5)$ & $484(56,4)$ & 294 \\
\hline $20 \mathrm{~min}$ & $838(71,8)$ & $1531(666,9)$ & $912(457,5)$ & $739(284,6)$ & 908 \\
\hline $30 \mathrm{~min}$ & $1277(410,8)$ & $2104(791,0)$ & $1130(637,8)$ & $1298(648,4)$ & 1228 \\
\hline $40 \mathrm{~min}$ & $1584(534,7)$ & $2328(779,2)$ & $1419(972,4)$ & $1715(823,3)$ & 1468 \\
\hline $50 \mathrm{~min}$ & $1838(453,6)$ & $2600(718,0)$ & $1635(1207,8)$ & $1931(883,5)$ & 1544 \\
\hline $60 \mathrm{~min}$ & $2290(336,5)$ & $2655(726,3)$ & $1813(1406,5)$ & $2155(996,3)$ & 1610 \\
\hline $75 \mathrm{~min}$ & $2872(187,4)$ & $2919(747,4)$ & $1922(1483,3)$ & $2521(1228,1)$ & 1858 \\
\hline $90 \mathrm{~min}$ & $3189(83,3)$ & $3198(662,7)$ & $2015(1551,4)$ & $2695(1310,6)$ & 1887 \\
\hline $105 \mathrm{~min}$ & $3495(245,7)$ & $3582(647,9)$ & $2382(1680,6)$ & $2813(1411,5)$ & 2314 \\
\hline $120 \mathrm{~min}$ & $3644(281,0)$ & $3699(690,6)$ & $2490(1706,3)$ & $3065(1553,0)$ & 2357 \\
\hline 2 h 30 & $4126(560,7)$ & $4331(650,6)$ & $2669(1829,4)$ & $3382(1718,7)$ & 2787 \\
\hline $3 \mathrm{~h}$ & $4584(801,1)$ & $4762(579,5)$ & $3112(1961,0)$ & $4063(2288,8)$ & 2926 \\
\hline 3 h 30 & $4998(1056,4)$ & $5307(657,5)$ & $3390(2109,3)$ & $4561(2404,4)$ & 3030 \\
\hline $4 \mathrm{~h}$ & $5823(1540,5)$ & $5839\{907,9\}$ & $3818(2504,5)$ & $4846(2528,9)$ & 3176 \\
\hline $4 \mathrm{~h} 30$ & $6219(1344,5)$ & $6248(1003,7)$ & $4222(2644,7)$ & $5051(2729,8)$ & 3277 \\
\hline $5 \mathrm{~h}$ & $6743(1132,8)$ & $7025(1014,0)$ & $4749(2970,8)$ & $5217(2762,4)$ & 3352 \\
\hline 5 h 30 & $7242(1372,0)$ & $7477(906,7)$ & $5165(3213,4)$ & $5454(2860,9)$ & 3383 \\
\hline $6 \mathrm{~h}$ & $7716(1829,6)$ & $8051(644,2)$ & $6231(3797,0)$ & $5937(3238,6)$ & 3407 \\
\hline 6 ค 30 & $8312(1591,3)$ & $8803(818,2)$ & $6564(3996,7)$ & $6146(3379,8)$ & 3415 \\
\hline $7 \mathrm{~h}$ & $8718(1669,6)$ & $9395(1002,2)$ & $6702(4042,0)$ & $6515(3543,7)$ & 3446 \\
\hline $\begin{array}{l}\text { Nbre de } \\
\text { repas }\end{array}$ & 3 & 4 & 3 & 3 & 1 \\
\hline
\end{tabular}




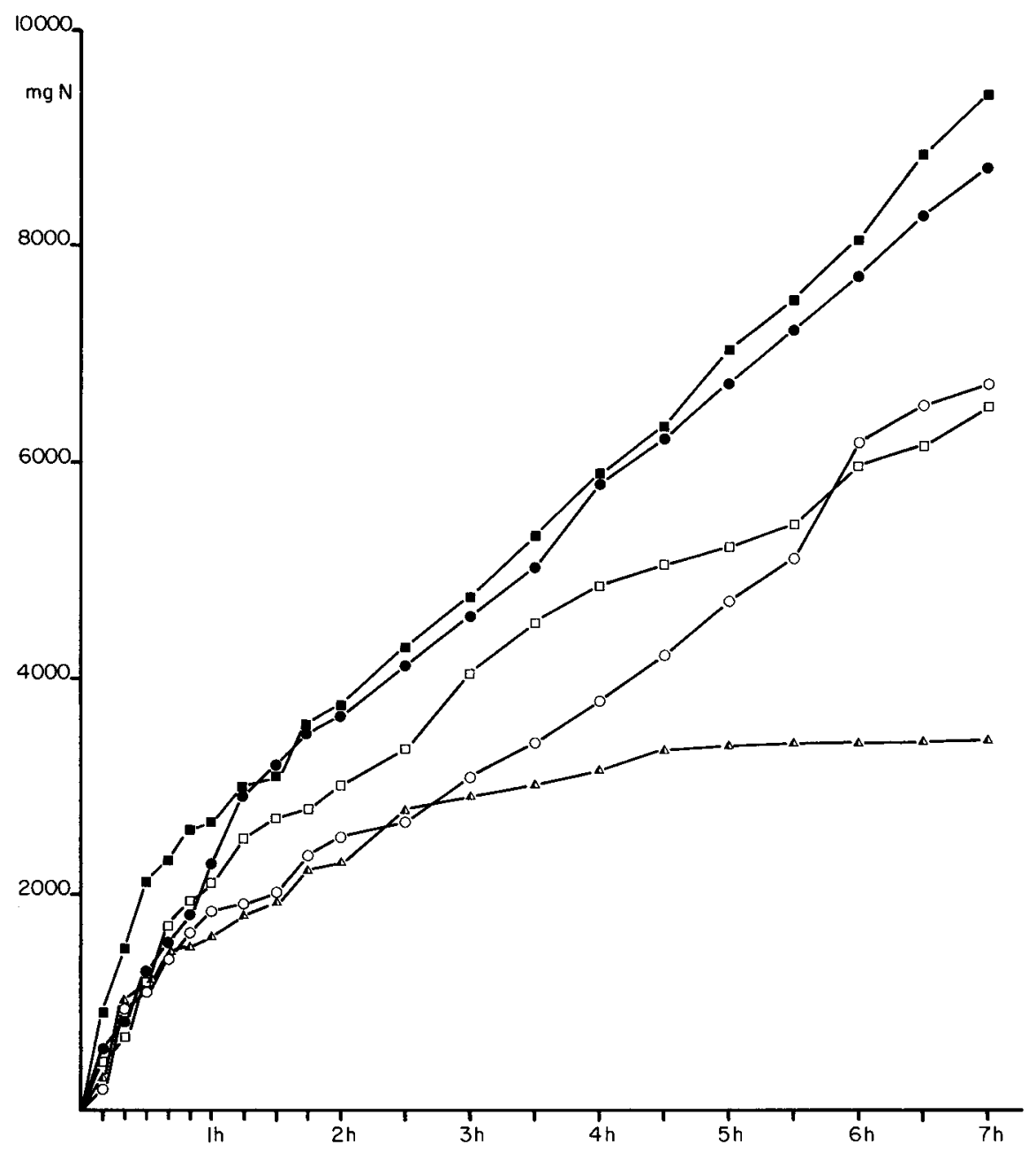

FIG. 3. - Evacuation gastrique de l'azote total (quantités cumulées par régime en $\mathrm{mg}$ ). Même légende que pour la figure 1.

cation. Les quantités totales d'azote recueilli en $7 \mathrm{~h}$ représentent, en moyenne, respectivement $33,36,29,29$ et $63 \%$ des quantités ingérées dans le cas des régimes $A, B, C, D$ et $E$.

L'analyse multivariate, effectuée avec les 20 mesures de quantité d'azote non protéique obtenues avec chacun des repas d'épreuve, laisse apparaître un comportement différent suivant le régime (fig. 4). Le pourcentage de NPN dans l'azote total (tabl. 2) augmente au cours du temps; avec les régimes " caséines ", il est plus faible qu'avec les régimes "laits", au cours des 2 ou 3 premières heures postprandiales mais devient plus élevé par la suite. Cependant, les résultats présentent une plus grande variabilité d'un repas d'épreuve à 


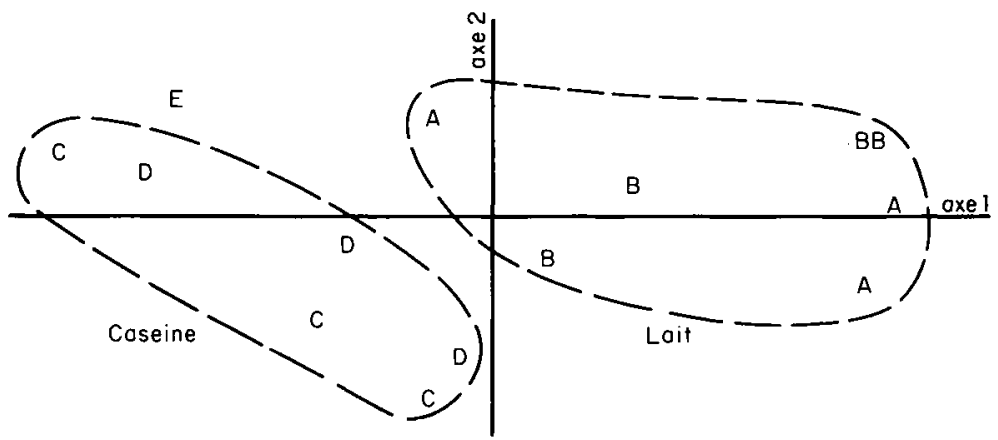

FIG. 4. - Analyse en composantes principales des quantités cumulées de NPN. Projection des 14 repas d'épreuves sur le plan principal 1-2. A : régime lait entier ; $B$ : régime lait écrémé ; $\mathrm{C}$ : régime caséine dans l'eau ; $\mathrm{D}$ : régime caséine dans un milieu minéral ; $\mathrm{E}$ : lactosérum.

TABLEAU 2

Evolution du pourcentage de NPN dans l'azote total (moyenne par régimes et écarts-types des échantillons)

\begin{tabular}{|c|c|c|c|c|c|}
\hline Durée & Régime A & Régime B & Régime $\mathrm{C}$ & Régime D & Régime $\mathrm{E}$ \\
\hline $10 \mathrm{~min}$ & $21,4(11,85)$ & $14,8(6,60\}$ & $11,3(3,12)$ & $9,7(0,30)$ & 15,9 \\
\hline $20 \mathrm{~min}$ & $21,5(8,58)$ & $15,1(3,96)$ & $11,3(9,92)$ & $8,7(1,92)$ & 12,5 \\
\hline $30 \mathrm{~min}$ & $22,7(7,30)$ & $16,8(2,87)$ & $11,3(10,04)$ & $8,8(4,35)$ & 12,2 \\
\hline $40 \mathrm{~min}$ & $22,2(5,97)$ & $17,2(2,80)$ & $11,5(10,30)$ & $8,7(4,45)$ & 12,2 \\
\hline $50 \mathrm{~min}$ & $21,4(4,37)$ & $17,4(2,62)$ & $13,0(12,12)$ & $9,0(5,22)$ & 12,3 \\
\hline $60 \mathrm{~min}$ & $19,8(3,92)$ & $17,5(2,53)$ & $13,9(12,18)$ & $10,2(5,82)$ & 12,3 \\
\hline 75 min & $19,4(3,01)$ & $17,7(1,95)$ & $14,9(12,68)$ & $11,6(6,27)$ & 12,5 \\
\hline $90 \mathrm{~min}$ & $19,9(3,19)$ & $18,4(1,87)$ & $16,0(12,80)$ & $13,1(6,82)$ & 12,5 \\
\hline $105 \mathrm{~min}$ & $20,5(3,17)$ & $18,6(1,76)$ & $20,6(11,69)$ & $13,7(6,54)$ & 11,1 \\
\hline $120 \mathrm{~min}$ & $20,9(2,93)$ & $18,9(1,66)$ & $22,0(12,60)$ & $15,3(6,89)$ & 12,7 \\
\hline 2 h 30 & $22,4(3,47)$ & $20,2(2,03)$ & $24,2(13,68)$ & $17,8(7,26)$ & 12,8 \\
\hline $3 \mathrm{~h}$ & $23,4(3,57)$ & $21,5(1,26)$ & $28,7(14,49)$ & $20,5(6,06)$ & 13,2 \\
\hline 3 h 30 & $23,8(3,56)$ & $22,7(1,57)$ & $30,4(14,60)$ & $24,9(8,69)$ & 13,8 \\
\hline $4 \mathrm{~h}$ & $25,3(4,18)$ & $24,4(2,07)$ & $32,2(14,20)$ & $26,3(9,78)$ & 14,4 \\
\hline $4 \mathrm{~h} 30$ & $26,2(3,21)$ & $25,1(1,76)$ & $33,3(14,21)$ & $26,9(9,41)$ & 15,1 \\
\hline $5 \mathrm{~h}$ & $27,2(2,48)$ & $25,6(2,14)$ & $35,1(13,15)$ & $28,0(10,30)$ & 15,5 \\
\hline 5 h 30 & $27,5(2,47)$ & $25,9(2,41)$ & $35,0(13,53)$ & $28,5(10,94)$ & 15,8 \\
\hline $6 \mathrm{~h}$ & $27,5 \backslash 2,32\}$ & $26,4(2,72)$ & $34,2(14,26)$ & $29,0(11,26)$ & 16,1 \\
\hline 6 h 30 & $26,7\{3,56\}$ & $26,4(2,63)$ & $34,5(14,26)$ & $29,4(11,19)$ & 16,1 \\
\hline $7 \mathrm{~h}$ & $26,7\langle 3,31\}$ & $26,1(2,92)$ & $34,8(14,52)$ & $29,2(11,53)$ & 16,4 \\
\hline $\begin{array}{l}\text { Nbre de } \\
\text { repas }\end{array}$ & 3 & 4 & 3 & 3 & 1 \\
\hline
\end{tabular}

l'autre avec les régimes " caséines " qu'avec les régimes « laits ». Dans le cas du régime $E$, le pourcentage de NPN augmente également au cours du temps, mais les valeurs obtenues sont plus faibles qu'avec les autres régimes. Afin de comparer les différents régimes entre eux, nous avons effectué une analyse en composantes principales pour l'ensemble des 14 repas d'épreuve, en prenant en compte les 4 variables suivantes : volume de matière fraîche, quantité de NPN, quantité d'azote protéique et pourcentage de NPN dans I'azote total. L'image ainsi obte- 
nue (fig. 5) permet de mettre en évidence deux types de comportement différents concernant, d'une part les régimes $A$ et $B$, et d'autre part les régimes $C$ et D. Pour les régimes "caséines", la dispersion des points suivant l'axe 2 de part et d'autre de l'axe principal est beaucoup plus importante que pour les deux " régimes lait " pour lesquels l'ensemble des points sont plus regroupés le long de I'axe 1 . Avec le régime $E$, les points semblent plus regroupés mais le faible nombre de mesures effectuées avec ce régime empêche toute conclusion définitive.

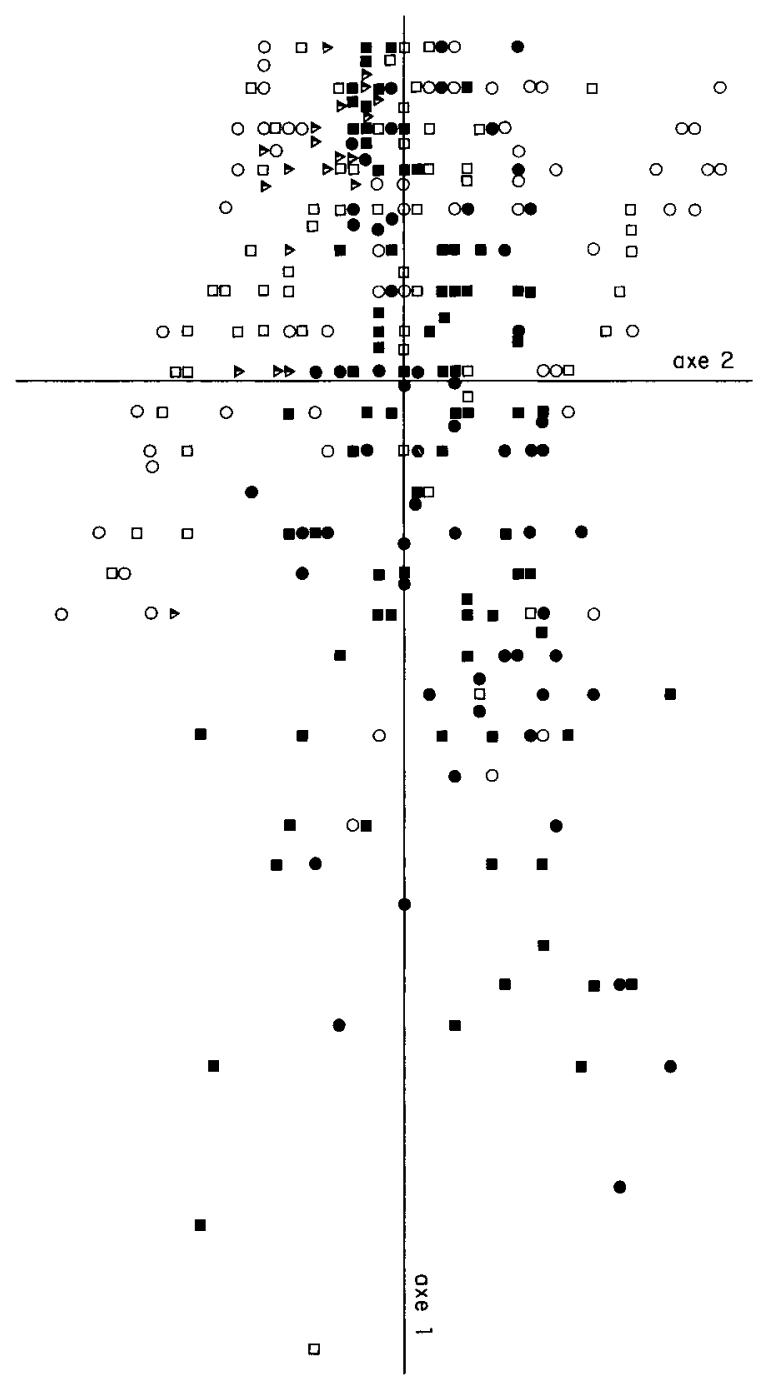

FIG. 5. - Analyse en composantes principales calculée en prenant en compte les quantités de matière fraîche de NPN et d'azote protéique ainsi que le pourcentage de NPN dans l'azote total. Même légende que pour la figure 1. 
La coagulation des caséines dans l'estomac modifie l'évacuation des protéines vers le duodénum. Comme ces protéines fixent fortement le calcium, il est intéressant de suivre avec les régimes " laits ", les régimes " caséine " ne contenant pas de calcium, la cinétique d'arrivée de cet élément dans l'intestin. Nos digesta étant collectés dans une solution de TCA à une concentration finale de $12 \%$, les ions $\mathrm{Ca}^{2+}$ doivent, en raison du pouvoir dissociant de ce milieu, se retrouver dans la fraction soluble. La quantité de calcium recueillie en $7 \mathrm{~h}$ dans les surnageants ne représente qu'une faible part du calcium ingéré (35\%) (fig. 6). Malgré une variabilité relativement faible (de l'ordre de $10 \%$ ) observée entre les 7 repas d'épreuve, aucune différence ne peut être détectée entre les régimes $A$ et $B$. D'autre part, si la quantité de calcium recueillie est corrélée au volume de produit quittant la caillette ( $p<2 \%$ ), elle est beaucoup plus liée à la quantité de NPN obtenue $(p \ll 1 \%)$.

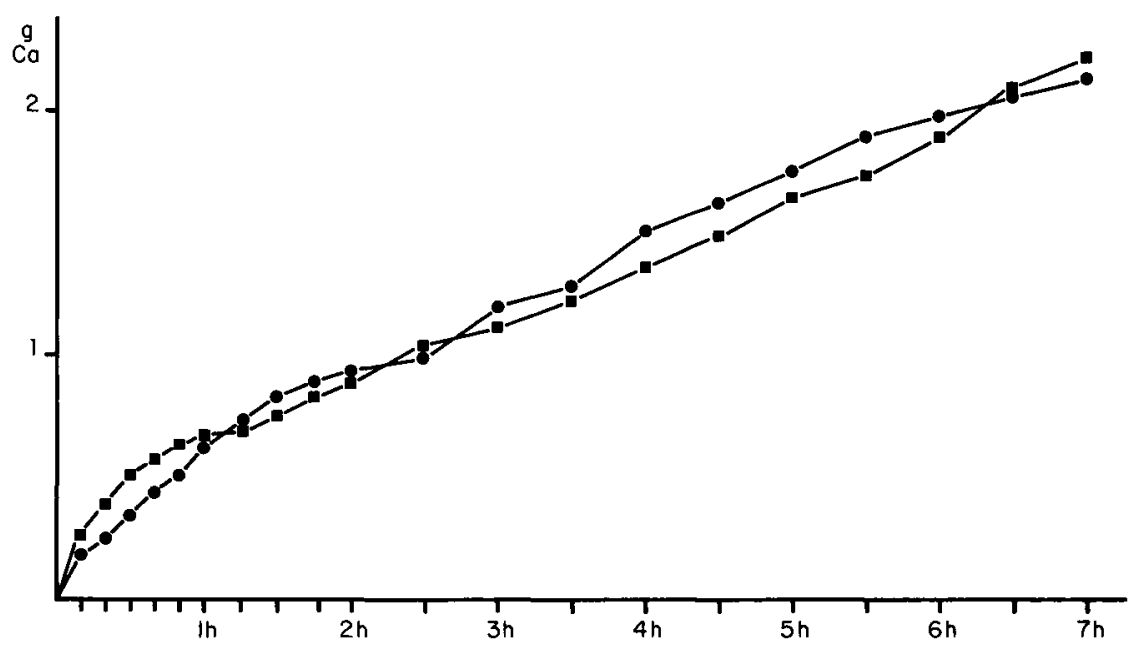

FIG. 6. - Evacuation gastrique du calcium avec les "régimes laits" (quantités cumulées en g). Même légende que pour la figure 1.

\section{Discussion.}

Les résultats font apparaître une variabilité importante dans les répétitions des repas d'épreuve, surtout avec les régimes "caséines ". Une variabilité du même ordre que celle obtenue avec les régimes " laits " a également été observée dans nos essais antérieurs, en étudiant l'évacuation gastrique de laits de remplacement contenant diverses sources de protéines (Toullec, Thivend et Mathieu, 1971 ; Guilloteau et al., 1975). Cela est vraisemblablement à relier au fait qu'après le repas, la motricité est désorganisée pendant un temps plus ou moins long selon l'animal ou le jour (Dardillat, 1982). Ce phénomène pourrait être accentué par la distribution de repas d'épreuve, surtout dans le cas des régimes " caséines", dont la composition s'écarte davantage de celle du lait de remplacement habituellement ingéré par les animaux. En conséquence, et à cause du nombre relativement restreint de répétitions qu'il a été possible d'effectuer 
avec un même régime ( 4 au maximum), aucune différence significative n'a pu être mise en évidence entre les régimes pour les paramètres mesurés (débit par période ou cumulé, quantité d'azote protéique et quantité de NPN) pris séparément. Cependant, l'évolution du pourcentage de NPN dans l'azote total (fig. 4) et l'analyse des résultats (fig. 5) indiquent un comportement différent des 2 régimes " laits » ( $A$ et $B$ ) par rapport aux 2 régimes " caséines » ( $C$ et $D$ ). La protéolyse des lactoprotéines et leur évacuation gastrique semblent donc différer suivant le régime. En particulier, les régimes "caséines " sont soumis à une protéolyse plus intense probablement permise par l'absence de coagulum qui rend les liaisons peptidiques plus accessibles aux protéases ; de plus, ces régimes ont un pouvoir tampon plus faible, au moins localement, ce qui favorise l'action protéolytique par l'obtention d'un $\mathrm{pH}$ mieux adapté aux enzymes gastriques. En revanche, les protéines du régime $E$ semblent être beaucoup moins attaquées dans la caillette. Cela serait dû à leur temps de séjour plus court et à leur sensibilité moins grande aux enzymes protéolytiques (Tourneur et Lenoir, 1980). Le régime $E$ n'a pu faire l'objet que d'un seul repas d'épreuve. Cependant, les différences observées par rapport aux régimes "laits" sont en accord avec celles déjà décrites pour l'évacuation gastrique de l'azote et du NPN, en comparant les aliments d'allaitement dont les protéines provenaient du lait ou du lactosérum (Toullec, Thivend et Mathieu, 1971; Guilloteau et Toullec, 1978).

Si la quantité de matière fraîche sortant de la caillette en $7 \mathrm{~h}$ est voisine de celle ingérée au cours du repas, par contre la quantité d'azote total contenue dans les digesta ne correspond qu'à une faible part (environ $30 \%$ ) de l'azote ingéré dans le cas des régimes $A, B, C$ et $D$. Ce résultat peut sembler à première vue surprenant. En effet, Guilloteau et al. (1975, 1979) collectaient en 7 h l'équivalent de $68 \%$ de l'azote ingéré chez des animaux munis du même type de canule, mais non mis à jeun avant le repas d'épreuve. Cependant, Ternouth et Roy (1973) en utilisant du lait dont les protéines contenaient de la lysine tritiée, n'obtenaient que $50 \%$ de la radioactivité ingérée au cours des 12 premières heures postprandiales. Cette valeur est en accord avec notre résultat de $30 \%$ en $7 \mathrm{~h}$. De même, les matières grasses sont étroitement associées aux caséines lors de la coagulation; or, Toullec (1968) a observé, chez le veau, le passage de quantités importantes d'acide laurique dans la lymphe du canal thoracique jusqu'à plus de $30 \mathrm{~h}$ après un repas d'épreuve apportant de I'huile de coprah, bien que deux repas d'épreuve dépourvus de cet acide aient eu lieu 12 et $24 \mathrm{~h}$ après. II semble donc que les produits entrant dans le duodénum chez un veau dont la caillette n'a pas été préaiablement vidée, correspondent pour une part seulement à l'aliment ingéré lors du dernier repas et qu'ils proviennent en proportion non négligeable des repas précédents. Les protéines devraient donc être davantage protéolysées que dans notre essai, du fait de leur temps de contact plus long avec les enzymes gastriques. Cette hypothèse explique les différences que nous observons par rapport aux résultats de Guilloteau et al. (1975) qui indiquaient un pourcentage de NPN de l'ordre de $50 \%$ de l'azote total dès l'ingestion du lait.

De plus, notre technique de collecte des échantillons peut influencer la vitesse d'évacuation gastrique des produits azotés. En effet, comme nous avons 
recueilli la totalité des produits quittant la caillette, afin de disposer de grandes quantités d'échantillons pour les analyses ultérieures, il nous a fallu réintroduire dans l'intestin de l'animal un liquide de composition suffisamment voisine de celle des digesta prélevés. Cet hydrolysat était introduit dans l'intestin à un débit aussi proche que possible de celle de l'évacuation gastrique. Cependant, si la vitesse d'arrivée du liquide dans l'intestin n'était pas trop modifiée par ce procédé, la nature des produits azotés, au lieu de suivre l'évolution au cours du temps de l'hydrolyse des lactoprotéines, correspondait à un hydrolysat moyen identique durant toute l'expérience. Cela pourrait peut-être également expliquer en partie la quantité relativement faible d'azote recueillie au cours de notre essai.

L'évacuation gastrique des protéines est moins rapide avec les régimes $A, B$, $C$ et $D$ qu'avec le régime $E$. Pour les régimes "laits », le ralentissement est dû à la coagulation ; pour les régimes "caséines", il est sans doute déterminé par la précipitation des protéines qui se produit quand le $\mathrm{pH}$ stomacal se rapproche de 4,6. Par rapport aux régimes " caséines », les régimes "laits » semblent entraîner l'arrivée dans le duodénum de quantités plus importantes d'azote, rapportées aux quantités ingérées. Les régimes "caséines" ne contiennent pas de protéines du lactosérum qui, n'étant pas précipitées, pourraient quitter la caillette rapidement. Cependant, si l'on additionne les quantités d'azote obtenues, par exemple au cours des 3 premières heures postprandiales avec les régimes " caséines » et le régime " lactosérum ", les valeurs obtenues correspondent respectivement à 21 et $25 \%$ des quantités ingérées avec $C+E$ et $D+E$, au lieu de 17 et $18 \%$ avec les régimes $A$ et $B$. La coagulation ralentirait donc davantage l'évacuation gastrique que ne le fait la précipitation des caséines sous l'effet de l'acidité de la caillette. Nos résultats sont en accord avec ceux de Frantzen et al. (1973) mais permettent de plus de souligner l'effet de la précipitation des caséines.

Avec les régimes "laits", la quantité de calcium recueillie représente également une part relativement faible (environ $35 \%$ ) de la quantité ingérée. Mais le calcium se fixe de manière importante sur les caséines et la coagulation de ces dernières retarde probablement l'arrivée de cet élément dans le duodénum. D'ailleurs, si la quantité de calcium recueillie est corrélée au volume de produit quittant la caillette $(p<2 \%)$, elle est beaucoup plus liée à la quantité de NPN obtenue $(P \ll 1 \%)$. Cela met en évidence l'importance de l'hydrolyse stomacale des protéines du lait sur la vidange du calcium. II est probable qu'au cours de cette hydrolyse est libéré un certain nombre de peptides susceptibles de fixer du calcium ; ces peptides, du fait de leur charge (phosphopeptides en particulier), n'interviennent que peu dans le maintien du coagulum dans l'estomac et sont donc libérés plus rapidement au cours de la digestion.

Cette première analyse des produits arrivant dans le duodénum durant les $7 \mathrm{~h}$ qui suivent l'ingestion d'un repas d'épreuve nous a permis de mettre en évidence, chez le jeune veau dont la caillette avait été préalablement vidée, une évacuation gastrique relativement lente. Si la coagulation du lait entraîne un net ralentissement de l'évacuation gastrique, la précipitation des caséines, sous l'effet de l'abaissement du $\mathrm{pH}$ de l'estomac, provoque un effet semblable mais moins accentué. En outre, la sortie du calcium semble être fortement liée à la 
présence de produits de dégradation des caséines qui, solubles en TCA $12 \%$, pourraient être des phosphopeptiodes. Une étude biochimique plus élaborée de ces échantillons devrait apporter des informations supplémentaires utiles pour mieux comprendre la protéolyse gastrique des lactoprotéines.

Reçu en février 1982.

Accepté en octobre 1982.

\section{Références}

ADDEO F., PÉLISSIER J.-P., CHIANESE L., 1980. Specific action of milk clotting enzymes on enzymes on water buffalo caseins. I. Effect of chymosin on $\beta$-casein. J. Dairy Res., 47, 421426.

ASH R. W., 1962. Gastro-intestinal reentrant cannulea for studies of digestion in sheep. Anim. Prod., 4, 309-312.

BRAUDE R., MITCHELL K. G., NEWPORT M. J., PORTER J. W. G., 1970 a. Artificial rearing of pigs. I. Effect of frequency and level of feeding on performance and digestion of milk proteins. Br. J. Nutr., 24, 501-516.

BRAUDE R., NEWPORT M. J., PORTER J. W. C., 1970 b. Artificial rearing of pigs. II. The time course of milk protein digestion and proteolytic enzyme secretion in the 28-day-old pig. Br. J. Nutr., 24, 827-842.

BURACZEWSKI S., PORTER J. W. G., ROLLS B. A., ZEBROWSKA T., 1971. The course of digestion of different food proteins in the rat. Br. J. Nutr., 25, 299-306.

CREAMER L. K., MILLS O. E., RICHARDS E. L., 1971. The action of rennets on the caseins. I. Rennin action on $\beta$-casein solution. J. Dairy Res., 38, 269-280.

DARDILLAT C., 1982. Communication personnelle.

DELFOUR A., JOLLES J., ALAIS C., JOLLES P., 1965. Caseino-glycopeptides : characterization of methionine residue and of the $\mathrm{N}$-terminal sequence. Biochem. Biophys. Res. Comm., 19. 452-455.

FOLTMANN B., JENSEN A., LONBLAD P., SMIDT E., AXELSEN N., 1981. A Developmental analysis of the production of chymosin and pepsin in pigs. Comp. Biochem. Physiol., 68B, 9-13.

FOX P. F., WALLEY B. F., 1971. Influence of sodium chloride on the proteolysis of casein by rennet and by pepsin. J. Dairy Res., 38, 165-170.

FRANTZEN J.-F., TOULLEC R., THIVEND P., MATHIEU C. M., 1973. Influence de la coagulation des protéines sur la vidange stomacale chez le veau préruminant. Ann. Biol. anim. Bioch. Biophys., 13, 718-721.

GUILLOTEAU P., PARUELLE J.-L., TOULLEC R., MATHIEU C.-M., $1975 . \quad$ Utilisation des protéines par le veau préruminant à l'engrais. III. Influence du remplacement des protéines du lait par celles du poisson sur la vidange stomacale. Ann. Zootech., 24, 243-253.

GUILLOTEAU P., TOULLEC R., SAUVANT D., PARUELLE J. L., 1979. Utilisation des protéines par le veau préruminant à l'engrais. VII. Influence du remplacement des protéines du lait par celles du soja ou de la féverolle sur l'évacuation gastrique. Ann. Zootech., 28, 1-17.

GUILLOTEAU P., TOULLEC R., 1978. Digestion des protéines du lactosérum dans la caillette du veau préruminant. $X X^{e}$ Congr. int. Laiterie, 942. Synaps, Neuilly.

HILL K. J., NOAKES D. E., LOWE R. A., 1970. Gastric digestive physiology of the calf and piglet, 166-179. In PHILLIPSON A. T., Physiology of digestion and metabolism in the ruminant. Oriel Press, Newcastle upon Tyne.

HILL R. D., LAHAV E., GIVOL D., 1974. A rennin sensitive bond in $\alpha_{\mathrm{s} 1} \beta$-casein. J. Dairy Res., 41, 147-153.

JENNESS R., KOOPS J., 1962. Preparation and properties of a salt solution which simulates milk ultrafiltrate. Neth. Milk Dairy J., 16, 153-164.

MIRANDA G., PÉLISSIER J.-P., 1981 a. In vivo studies on the digestion of bovine caseins in the rat stomach. J. Dairy Res., 48, 319-326. 
MIRANDA G., PELISSIER J.-P., 1981 b. Etude de la protéolyse in vivo des caséines bovines dans l'estomac du rat. Reprod. Nutr. Dévelop., 21, 861.

MIRANDA G., PÉLISSIER J.-P., 1982. Kinetic studies of in vivo digestion of bovine skim-milk proteins in the rat stomach. J. Dairy Res. (sous presse).

PÉLISSIER J.-P., MERCIER J.-C., RIBADEAU-DUMAS B., 1974. Etude de la protéolyse des caséines $\alpha_{\mathrm{s} 1}$ et $\beta$ bovines par la présure. Spécificité d'action. Peptides amers libérés. Ann. Biol. anim. Bioch. Biophys., 14, 343-362.

TERNOUTH J. H., ROY J. H. B., 1973. The effect of diet and feeding technique on digestive function in the calf. Ann. Rech. vet., 4, 19-30.

TOULLEC R., 1968. Absorption des matières grasses par la voie lymphatique chez le veau préruminant. Ann. Biol. anim. Bioch. Biophys., 8, 445-447.

TOULLEC R., THIVEND P., MATHIEU C.-M., 1971. Utilisation des protéines du lactosérum par le veau préruminant à l'engrais. I. Vidange stomacale comparée du lait entier et de deux laits de remplacement ne contenant que des protéines de lactosérum comme source de matières azotées. Ann. Biol. anim. Bioch. Biophys., 11, 435-453.

TOURNEUR C., LENOIR J., 1980. Sensibilité in vitro des protéines du lactosérum à l'hydrolyse enzymatique. Ann. Nutr. Alim., 34, 42-43.

VISSER S., 1981. Proteolytic enzymes and their action on milk proteins. A review. Neth. Milk Dairy J., 35, 65-88.

VISSER S., SLANGEN K. J., 1977. On the specificity of chymosin (rennin) in its action on bovine $\beta$-casein. Neth. Milk Dairy J., 31, 16-30.

ZEBROWSKA T., 1968. The course of digestion of different food proteins in the rat. Fractionation of the nitrogen in intestinal contents. Br. J. Nutr., 22, 483-491. 